\title{
Síndrome de Eagle com remoção cirúrgica do processo estiloide calcificado: Relato
}

\section{de caso clínico}

\author{
Eagle Syndrome with surgical removal of the calcified styloid process: A clinical case report \\ Síndrome de Eagle con remoción quirúrgica de la apófisis estiloides calcificada: Reporte de caso \\ clínico
}

Recebido: 11/06/2021 | Revisado: 18/06/2021 | Aceito: 19/06/2021 | Publicado: 04/07/2021

Thaisa Reis de Carvalho Sampaio

ORCID: https://orcid.org/0000-0002-4345-3662

Universidade de Pernambuco, Brasil

E-mail: thaisa.sampaio@upe.br

André Coelho Lopes

ORCID: https://orcid.org/0000-0002-9587-1137

Universidade de Pernambuco, Brasil

E-mail: andre.coelho@upe.br

Marcelo Marotta Araújo

ORCID: https://orcid.org/0000-0002-7295-0780

Universidade Estadual Paulista, Brasil

E-mail: drmarceloaraujo@hotmail.com

Kayo Costa Alves

ORCID: https://orcid.org/0000-0002-7788-820X

Centro Universitário Mario Pontes Jucá, Brasil

E-mail: kayocostaalves@gmail.com

Ivan José Moreira Oliveira

ORCID: https://orcid.org/0000-0002-8198-8801

Universidade Estadual Paulista, Brasil

E-mail: ivan.oliveira@me.com

Bruno da Silva Mesquita

ORCID: https://orcid.org/0000-0002-2824-4088

Universidade de Pernambuco, Brasil

E-mail: bruno.smesquita@upe.br

Francisco Paulo Araujo Maia

ORCID: https://orcid.org/0000-0002-3137-0173

Universidade de Pernambuco, Brasil

E-mail: francisco.maia@upe.br

Damião Edgleys Porto

ORCID: https://orcid.org/0000-0002-7000-7887

Universidade de Pernambuco, Brasil

E-mail: edgleys.porto@upe.br

Emanuel Savio de Souza Andrade

ORCID: https://orcid.org/0000-0003-2165-4217

Universidade de Pernambuco, Brasil

E-mail: emanuel.savio@upe.br

\section{Resumo}

A síndrome de Eagle é uma condição clinica associada a um processo estiloide aumentado, com mais de $25 \mathrm{~mm}$ de comprimento, o que gera uma compressão de estruturas anatômicas importantes provocando sintomas como dor cervical, dor facial, disfagia, otalgia, limitação de abertura bucal e outros. Por se tratar de uma síndrome rara com sintomas semelhantes a outras condições patológicas, é importante a realização do correto diagnóstico e tratamento. Seu tratamento pode ser conservador ou cirúrgico pela abordagem intra ou extra-oral. Este estudo trata-se de um relato de caso clinico, de um paciente 40 anos de idade, do gênero masculino, diagnosticado com síndrome de Eagle após apresentar sintomatologia dolorosa à abertura de boca, disfagia e otalgia e exame tomográfico evidenciar o processo estiloide com $30 \mathrm{~mm}$ de comprimento. O tratamento de escolha foi a excisão cirúrgica do processo estiloide através da abordagem extra-oral, garantindo maior controle dos grandes vasos, melhor exposição e menor risco de contaminação bacteriana dos espaços cervicais profundos. Como resultado, o tratamento de escolha foi bem sucedido, com regressão total da sintomatologia, sem complicações.

Palavras-chave: Processo mastoide; Ossificação heterotópica; Anormalidades maxilofaciais.

\section{Abstract}

Eagle syndrome is a clinical condition associated with an increased styloid process, reaching more than $25 \mathrm{~mm}$ in length, which generates a compression of crucial anatomical structures, causing neck and facial pain, dysphagia, 
otalgia, mouth opening limitation and other symptoms. Being a rare syndrome with symptoms similar to other pathological conditions, it is essential to implement the correct diagnosis and treatment. The treatment can be conservative or surgical by intraoral or extraoral approach. This study is a clinical case report, of a 40-year-old male subject, diagnosed with Eagle syndrome after presenting painful symptoms on mouth opening, dysphagia and otalgia and tomographic examination showing the styloid process with $30 \mathrm{~mm}$ of length. The chosen treatment was surgical excision of the styloid process using the extra-oral approach, ensuring greater control of the great vessels, better exposure and less risk of bacterial contamination of the deep cervical spaces. As a result, the treatment was successful, with complete regression of symptoms without complications.

Keywords: Eagle syndrome; Styloid process; Mouth opening.

\section{Resumen}

El síndrome de Eagle es una condición clínica asociada con un aumento de la apófísis estiloides, de más de $25 \mathrm{~mm}$ de largo. Esto genera una compresión de importantes estructuras anatómicas causando síntomas como dolor de cuello, dolor facial, disfagia, otalgia, limitación de la apertura de la boca y otros. Como es un síndrome poco común con síntomas similares a otras afecciones, es importante realizar un correcto diagnóstico y tratamiento. El tratamiento puede ser conservador o quirúrgico por abordaje intra o extraoral. Este estudio describe el caso de un hombre de 40 años de edad que fue diagnosticado con síndrome de Eagle después de presentar dolor al abrir la boca, disfagia, otalgia y tomografía que muestra la apófisis estiloides de $30 \mathrm{~mm}$ de largo. El tratamiento de elección fue la excisión quirúrgica de la apófisis estiloides por abordaje extraoral, asegurando un mayor control de los grandes vasos, una mejor exposición y un menor riesgo de contaminación bacteriana de los espacios cervicales profundos. Como consecuencia, el tratamiento de elección fue exitoso, con regresión completa de los síntomas, sin complicaciones.

Palabras clave: Apófisis mastoides; Osificación heterotópica; Anomalías maxilofaciales.

\section{Introdução}

O processo estiloide é uma projeção do osso temporal que mede cerca de $25 \mathrm{~mm}$, nele estão inseridos os músculos estilo-hioideo, estiloglosso e estilo-faríngeo. Medialmente a esse processo localizam-se estruturas relevantes como veia jugular interna, artéria carótida externa e interna, os nervos vago, glossofaríngeo, acessório e hipoglosso (Pigache et al., 2018).

Descrito pela primeira vez por um otorrinolaringologista chamado Eagle em 1937 (Eagle, 1937), a síndrome de Eagle é caracterizada por um alongamento ósseo ou uma calcificação do ligamento do processo estiloide, podendo este chegar até 50mm de comprimento (Eagle, 1948; 1949). Os sintomas decorrentes deste alongamento são disfagia, otalgia, disgeusia e dores cervicais, principalmente à abertura bucal e, em alguns casos, essa síndrome pode ser assintomática (Eagle, 1958; Beder, Ozgursoy \& Karatayli, 2005; Bedi et al., 2019; Scharukh, Basem \& Gregory, 2017; Saccomanno et al., 2018). Seu diagnóstico é realizado através dos sintomas juntamente com um exame radiográfico, como laterais oblíquas de mandíbula, radiografia panorâmica ou exame tomográfico (De Ferreira et al., 2003; Czajka et al., 2019).

O tratamento da síndrome de Eagle pode ser conservador, ou seja, acompanhar a evolução e o uso de medicação quando necessário, ou tratamento cirúrgico (Ceylan et al., 2008), somente indicado quando o tratamento conservador não mascarar os sintomas, consiste em uma Estiloidectomia, ou seja, remoção do processo estiloide através de um acesso extra ou intra-oral (Chase et al., 1986). No acesso intra-oral, apesar de não ter cicatriz, existe um risco maior de infecção dos espaços cervicais devido a contaminação do meio bucal e uma possível injuria vascular (Chase et al., 1986). O acesso extraoral utilizado é o retro-mandibular, que permite melhor visualização da área, porém este corre risco de lesar alguns ramos do nervo facial, glossofaríngeo, hipoglosso, carótida externa, carótida interna e jugular interna (Ceylan et al., 2008; Chase et al., 1986).

O objeto deste trabalho é apresentar a remoção cirúrgica do processo estiloide calcificado unilateral através de acesso extraoral em um paciente portador da síndrome de Eagle.

\section{Metodologia}

Trata-se de um estudo de caso clinico qualitativo descritivo de um único paciente (Yin, 2015), onde foi relatado os métodos de diagnóstico e tratamento de uma condição sindrômica em questão, o resultado deste tratamento e sua discussão com os achados na literatura. O paciente assinou o termo de consentimento livre e esclarecido e esteve ciente da divulgação 
das imagens tomográficas e dos procedimentos cirúrgicos realizados para enriquecimento da comunidade científica. Não houve necessidade de submissão ao comitê de ética, levando em consideração que foi um estudo de caso isolado.

Paciente M.C.S., de 40 anos, do gênero masculino, leucoderma, compareceu ao ambulatório de cirurgia com queixa álgica à mastigação. À anamnese o mesmo referiu dor em região retro-mandibular à abertura bucal, disfagia e otalgia à movimentos mastigatórios, negando trauma em região. O paciente afirma já ter sido operado previamente o lado direito da mandibula. Ao exame físico nota-se presença de abertura bucal limitada, oclusão estável e dor à palpação em região retromandibular. Ao exame tomográfico nota-se o processo estiloide direito com dimensão dentro da normalidade e um alongamento do processo estiloide esquerdo com H.D de síndrome de Eagle (Figura 1).

Figura 1: Reconstrução tridimensional da tomografia de crânio, evidenciando o alongamento do processo estiloide do lado esquerdo.

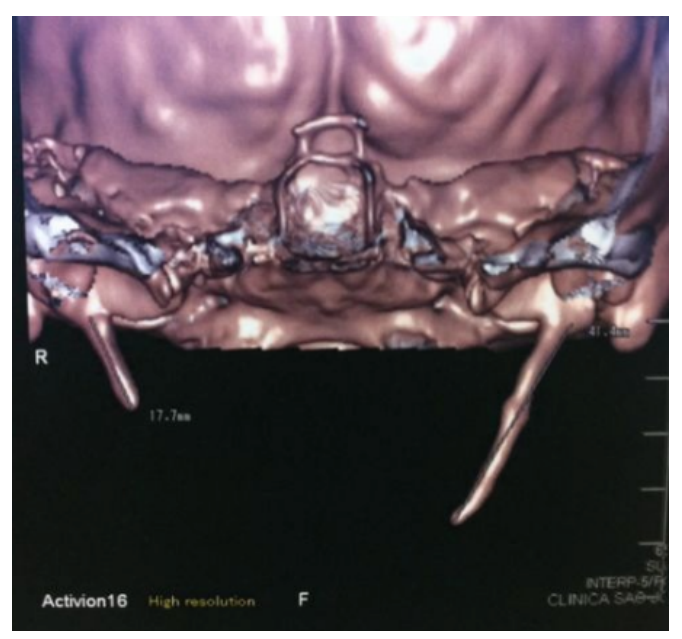

Fonte: Autores.

O procedimento cirúrgico foi realizado no hospital Policlin em centro-cirúrgico sob anestesia geral, o acesso de escolha foi extra-oral, realizado em região retro-mandibular com extensão inferior e o processo estiloide foi removido através de osteotomia sem intercorrência, após reflexão do periósteo e das inserções musculares. (Figura 2), o fragmento possuía 30mm (Figura 3). Ao acompanhamento ambulatorial nota-se ausência de sintomatologia dolorosa ao alimentar-se e à abertura bucal. 
Figura 2: Visão direta do processo estiloide alongado através da abordagem cirurgia extra-oral retro mandibular.

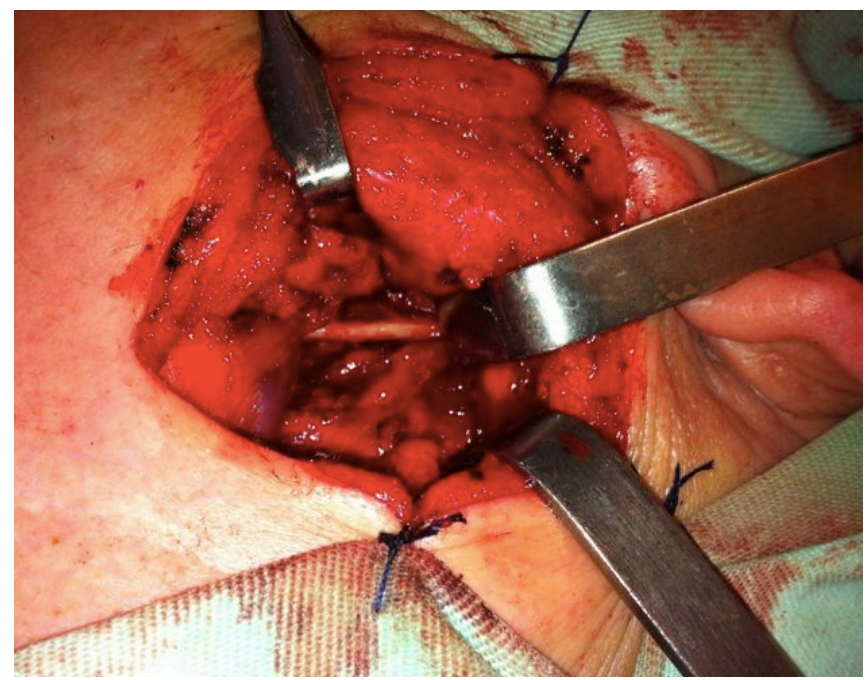

Fonte: Autores.

Figura 3: Fragmento do processo estiloide removido com $30 \mathrm{~mm}$ de comprimento.

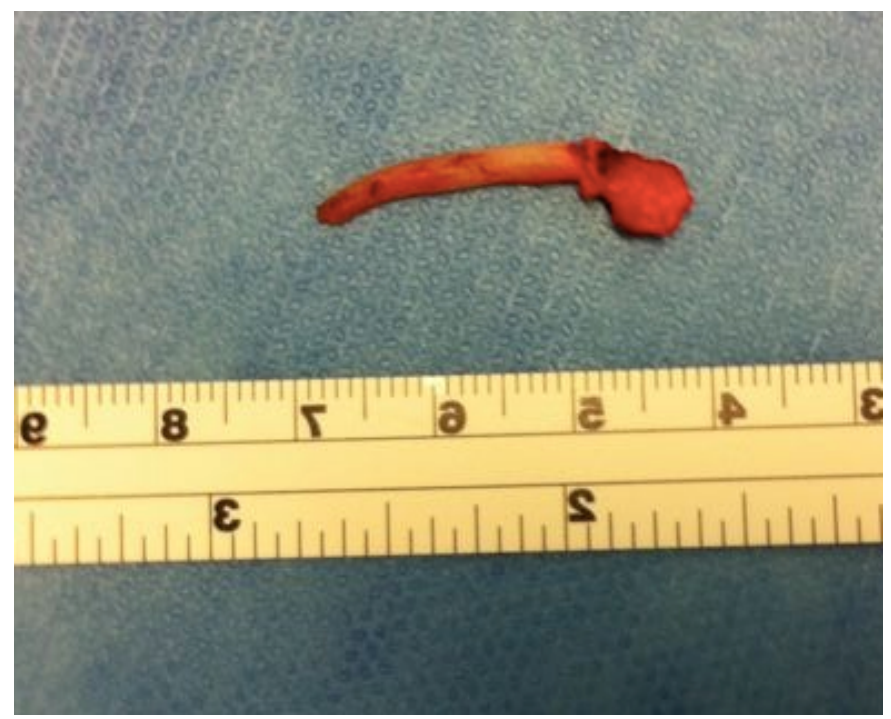

Fonte: Autores.

\section{Resultados e Discussão}

O processo estiloide normalmente mede aproximadamente $25 \mathrm{~mm}$ de acordo com a literatura. Ele é considerado alongado quando seu comprimento é maior que 25mm (Ceylan et al., 2008; Scharukh, Basem \& Gregory, 2017). No presente caso clinico, o processo estiloide apresentava $30 \mathrm{~mm}$ de comprimento. Ele reside em uma área anatomicamente complexa, e o seu aumento pode afetar estruturas vizinhas importantes. Se estende anteromedialmente a partir do processo mastoide e fica entre as artérias carótidas externa e interna. É o ponto de inserção dos músculos estiloglosso, estilo-hióideo, e estilofaríngeo e dos ligamentos estilo-hióideo e estilomandibular. Posteriormente ao processo estiloide está o forame estilomastoide, por onde saem o nervo facial e a artéria estilomastoide, lateral ao forame estilomastoideo está a sutura timpanomastoide, que abriga o ramo auricular do nervo vago. Medial ao processo estiloide está a artéria carótida interna, a veia jugular interna, o acessório espinhal e os nervos hipoglosso e glossofaríngeo e lateralmente à ponta estiloide está a artéria carótida externa e medialmente a ela está o músculo constritor superior e a fáscia faringobasilar (Scharukh, Basem \& Gregory, 2017; Pigache et al., 2018; 
Westbrook, Kabbaz \& Showalter, 2020).

A etiologia do que causa seu alongamento e consequentemente seus sintomas é obscura na literatura. A incidência de processo estiloide alongado foi relatada entre $1,4 \%$ a $30 \%$ da população, sua maioria com mais de 30 anos, e dentre eles, apenas 7,8\% a 10,3\% dos pacientes apresentam sintomas. As mulheres são afetadas com mais frequência do que os homens, em uma proporção de 3: 1. (Ceylan et al., 2008; Scharukh, Basem \& Gregory, 2017; Bedi et al., 2019; Waters et al., 2018; Badheya et al., 2017; Mahmoud \& Ashour, 2020)

Acredita-se que seu crescimento, provoca pressão nas estruturas vizinhas, como nervos e artérias e tonsilas, o que pode gerar sintomas neurais, vasculares e autonômicos como dor facial recorrente, cefaleia, disfagia, disfonia, restrição dos movimentos cervicais e sensação de corpo estranho na garganta (Grossmann \& Paiano, 1998; Scharukh, Basem \& Gregory, 2017; Westbrook, Kabbaz \& Showalter, 2020; Mahmoud \& Ashour, 2020; Saccomanno et al., 2018) No estudo de Waters et al. (2018), o comprimento estiloide mais longo foi correlacionado com escores aumentados para disfagia e odinofagia. De forma menos comum, já forma encontrados relatos de ataques isquêmicos transitórios reversíveis, consistindo em sintomas oftálmicos e episódios de pré-síncope devido a compressão da artéria carótida e diminuição do fluxo sanguíneo na artéria cerebral medial (Scharukh, Basem \& Gregory, 2017; Mahmoud \& Ashour, 2020).

No presente estudo, os sintomas encontrados foram dor em região retro-mandibular à abertura bucal, disfagia e otalgia à movimentos mastigatórios. O diagnóstico da síndrome de Eagle requer conhecimento da anatomia e um diagnóstico diferencial de exclusão. É necessário descartar outras condições como disfunção temporomandibular, otalgia, nevralgia, terceiros molares inclusos e lesão neoplásica, levando em consideração que os sintomas podem ser semelhantes. Uma radiografia panorâmica pode diagnosticar um processo estiloide alongado assim como a tomografia computadorizada, que é considerada o padrão ouro para o diagnóstico (Beder, Ozgursoy \& Karatayli, 2005; Scharukh, Basem \& Gregory, 2017; Mahmoud \& Ashour, 2020). O diagnóstico do caso em questão foi realizado após um exame físico detalhado dos sintomas, da palpação digital na fossa tonsilar e complementado por tomografia computadorizada, onde foi evidenciado o alongamento do processo estiloide e a exclusão das demais condições.

O tratamento da síndrome de Eagle pode ser clínico ou cirúrgico. O manejo clinico inclui repouso mandibular, dieta pastosa, tratamento medicamentoso com anti-inflamatórios e injeção tonsilar de corticosteroides. Quando não se obtém melhora após o tratamento clinico, se indica o tratamento cirúrgico, onde sua abordagem pode ser intra-oral ou extraoral (Bedi et al., 2019; Scharukh, Basem \& Gregory, 2017; Badheya et al., 2017; Czajka et al., 2019).

A abordagem intra-oral tem como vantagens, a ausência de incisão extraoral e como desvantagem a falta de controle dos vasos principais do pescoço, a exposição cirúrgica inadequada e a possibilidade de contaminação bacteriana dos espaços cervicais profundos. Para Al Weteid e Miloro (2015), em acessos intra-orais, o auxílio endoscópico evita complicações porém necessita de uma curva de aprendizado. Já durante a abordagem extra-oral, é possível obter o controle dos grandes vasos, melhor exposição e menor risco de contaminação bacteriana dos espaços cervicais profundos. Além disso, ela permite que um comprimento maior do estiloide seja removido do que seria possível por meio de uma abordagem intra-oral, e como desvantagens dessa técnica temos o maior tempo cirúrgico e a incisão extra-oral (Pigache et al., 2018; Czajka et al., 2019). Ambas as abordagens cirúrgicas fornecem o resultado desejado com resolução dos sintomas de acordo com a literatura, a escolha vai depender da experiência do cirurgião e vontade do paciente (Scharukh, Basem \& Gregory, 2017), no estudo de Czajka et al. (2019), houve resultado estatisticamente positivo para a melhora da dor independentemente da abordagem utilizada no procedimento cirúrgico.

O tratamento de escolha no presente caso clinico foi o tratamento cirúrgico com exérese do processo estiloide alongado através de acesso extra-oral assim como descrevem Ceylan et al. (2008), Chase et.al (1986) e Scharukh et al. (2017) pois este acesso evita contaminação e permite melhor visualização da área. De acordo com Kiraji et al. (2015), o tamanho do 
processo estiloide é decisivo na escolha do acesso a ser utilizado. Dessa maneira foi utilizado acesso extra-oral devido ao tamanho do fragmento a ser removido, a possibilidade de maior campo cirúrgico e a restrição de equipamentos endoscópicos.

\section{Considerações Finais}

Por se tratar de uma patologia rara, com sintomas semelhantes a outras condições patológicas, a síndrome de Eagle pode ser facilmente confundida, impedindo a realização do tratamento correto. Cabe ao médico ou dentista responsável, estar sempre atualizado e utilizar recursos, como exames de imagem, para diagnosticar e tratar corretamente a condição. O diagnóstico deverá ser feito através de um exame clínico detalhado com correto conhecimento da anatomia, juntamente com radiografia panorâmica ou tomografia computadoriza. O tratamento para a síndrome de Eagle com grande taxa de sucesso é a excisão cirúrgica do processo estiloide aumentado, onde a abordagem extra-oral mostrou sucesso no tratamento, sem complicações associadas e regressão total dos sintomas como foi observado no presente caso.

\section{Referências}

Al Weteid, A. S. \& Miloro, M. (2015). Transoral endoscopic-assisted styloidectomy: How should Eagle syndrome be managed surgically? Int $J$ Oral Maxillofac Surg, 44(9), 1181-1187. 10.1016/j.ijom.2015.03.021.

Badhey, A., Jategaonkar, A., Anglin, K. A. J., Kadakia, S., De Deyn, P. P., Ducic, Y., Schantz, S. \& Shin, E. (2017). Eagle syndrome: A comprehensive review. Clin Neurol Neurosurg, 159, 34-38. 10.1016/j.clineuro.2017.04.021.

Beder, E., Ozgursoy, O. B. \& Karatayli, O. S. (2005). Current diagnosis and transoral surgical treatment of Eagle’s syndrome. J Oral Maxillofac Surg, 63, $1742-1745$.

Bedi R. S, Aurora J. K., Chauhan, H., \& Komal, A. (2019). Eagle's Syndrome Mimicking Dental Pain: A Case Report With A Novel Surgical Approach. Natl J Maxillofac Surg, 10(2), 253-256. 10.4103/Njms.Njms_73_18.

Ceylan, A., Koybasioglu, A., Celenk, F., Yilmaz, O. \& Uslu, S. (2008). Surgical treatment of elongated styloid process: experience of 61 cases. Skull Base, 18, 289-295.

Chase, D. C., Zarmen, A., Bigelow, W. C. \& McCoy, J. M. (1986). Eagle's syndrome: a comparison of intraoral versus extraoral surgicalapproaches. Oral Surg Oral Med Oral Pathol, 62, 625-629.

Czajka, M., Szuta, M., Zapała, J. \& Janecka, I. (2019). Assessment of surgical treatment of Eagle's syndrome. Otolaryngol Pol, 73(5):18-24. 10.5604/01.3001.0013.1533.

De Ferreira, A. R. Jr, Muller, K., Hotta, T. H. \& Goncalves, M. (2003). Temporomandibular disorder or Eagle's syndrome? A clinical report. J Prosthet Dent, $90,317-320$.

Eagle, W. (1937). Elongated styloid process: report of two cases. Arch Otolaryngol, 25, 584-587.

Eagle, W. (1948). Elongated styloid process: Further observation and a new syndrome. Arch Otolaryngol, 47, 630-640.

Eagle, W. (1949). Symptomatic elongated styloid process: report of two cases of styloid process-carotid artery syndrome with operation. Arch Otolaryngol, 49, 490-503.

Eagle, W. (1958). Elongated styloid process: symptoms and treatment. AMA Arch Otolaryngol, 67, 172-176.

Grossmann, E. \& Paiano, G. A. (1998). Eagle's syndrome: a case report. Cranio Apr, 16(2), 126-30.

Kiraji, A., Illic, M., Pejakovic, B., Markov, B., Mijatov, S. \& Mijatov, I. (2015). Eagle's syndrome: A report of two cases. Vojnosanit Pregl, 72(5), 458-62.

Mahmoud, N. R. \& Ashour, E. M. (2020). Cervico-facial pain associated with Eagle's syndrome misdiagnosed as cranio-mandibular disorders. A retrospective study. J Craniomaxillofac Surg, 48(10), 1009-1017. 10.1016/j.jcms.2020.07.016.

Pigache, P., Fontaine, C., Ferri, J. \& Raoul, G. Transcervical styloidectomy in Eagle's syndrome. (2018). Eur Ann Otorhinolaryngol Head Neck Dis, 135, 433-436. https://doi.org/10.1016/j.anorl.2018.05.001.

Saccomanno, S., Greco, F., DE Corso, E., Lucidi, D., Deli, R., D'Addona, A. \& Paludetti, G. (2018). Eagle's Syndrome, from clinical presentation to diagnosis and surgical treatment: a case report. Acta otorhinolaryngologica Italica: organo ufficiale della Societa italiana di otorinolaringologia e chirurgia cervicofacciale, 38(2), 166-169. https://doi.org/10.14639/0392-100X-1479.

Scharukh, J., Basem, T. J. \& Gregory, A. G. (2017). Surgical Management of Long-standing Eagle's Syndrome. Ann Maxillofac Surg, 7(2), 232-236. 10.4103/ams.ams_53_17.

Yin, R. K. (2015). O estudo de caso. Bookman 
Research, Society and Development, v. 10, n. 8, e1610817096, 2021

(CC BY 4.0) | ISSN 2525-3409 | DOI: http://dx.doi.org/10.33448/rsd-v10i8.17096

Waters, C. M., Ho, S., Luginbuhl, A., Curry, J. M. \& Cognetti, D. M. (2018) Surgical Management of Stylohyoid Pain (Eagle's) Syndrome: A 5-Year Experience. Ann Otol Rhinol Laryngol, 128(3), 220-226. 10.1177/0003489418816999.

Westbrook, A. M., Kabbaz, V. J. \& Showalter, C. R. (2020). Eagle's syndrome, elongated styloid process and new evidence for pre-manipulative precautions for potential cervical arterial dysfunction. Musculoskelet Sci Pract, 50, 102219. https://doi.org/10.1016/j.msksp.2020.102219. 